\title{
Consultas terapéuticas: fenómenos curativos y salud*1
}

\section{Therapeutic consultations: healing phenomena and health}

Rosa Maria Tosta*2

\begin{abstract}
En este trabajo se relacionan los conceptos de salud y de los fenómenos curativos de D. W. Winnicott con su propuesta de Consultas Terapéuticas, modalidad de atención que activa la creatividad de la dupla psicoanalista y paciente. La idea asume que el ser humano tiene capacidades que emergen incluso en situaciones muy críticas que atraviesan la existencia humana. En las consultas es típico que el paciente ponga al terapeuta en una posición especial dentro de su imaginario, posibilitando un tipo de relación subjetiva diferente de la transferencia que se efectúa en un proceso analítico tradicional.
\end{abstract}

Palabras clave: Consulta terapéutica, fenómenos curativos, salud, creatividad

*1 Artículo compilado a partir de trabajo en apresetado en el XXV Encuentro Latinoamericano sobre el Pensamiento de D.W. Winnicott: Noviembre, 2016. Santiago de Chile. Ayuda PIPEq- PUC/SP.

*2 Pontifícia Universidade Católica de São Paulo - PUC-SP (São Paulo, SP, Br). 


\title{
ARTIGOS
}

\section{Introducción}

\author{
Es un juego sofisticado de esconder en el que es una \\ alegría estar escondido, pero un desastre no ser encontrado.
}

(Winnicott, 1963/1990, p. 169).

A partir del estudio del concepto de fenómenos curativos pude tener una nueva mirada a la práctica de consultas terapéuticas de Winnicott. Sólo pensando en el poder potencial de curación de cada ser humano mínimamente saludable es que podemos entender el éxito alcanzado en las consultas y no asignárselo a algún don mágico de Winnicott, aunque no podemos dejar de subrayar su real y viva presencia, su creatividad, madurez y capacidad clínica. Tales condiciones dicen un poco que cada uno - paciente y terapeuta necesitan aportar a la situación clínica. Del paciente, además de su angustia y sufrimiento, trae el potencial para la salud y la creatividad, revelados por su poder de curación; y del psicoanalista: su capacidad de estar presente y vivo en el encuentro humano, potenciando su poder para cuidar del otro y el poder de auto curación del paciente.

La creatividad es el denominador común a los dos participantes del encuentro, y la situación puede ser vista como transicional o constituyendo un espacio potencial. De hecho, esta práctica de la madurez de nuestro autor puede recoger de forma exuberante sus principales conceptos originales.

\section{Salud}

La esperanza de éxito viene de momentos de éxito relacionales, vividos tempranamente, que se constituyen en un almacenamiento de salud psíquica. Repetidas experiencias positivas de este encuentro primitivo proporcionarán una reserva de confianza en el mundo, o un quantum de salud.

Sabemos que la confianza o desconfianza en uno mismo y en el mundo se forma tempranamente en la vida. Al principio, cuando hay una dependencia 
casi absoluta del bebé con relación al cuidador y hay una adaptación materna suficientemente buena, lo que es registrado por el bebé no es el entorno, ya que no tiene condiciones de desarrollo para acceder a él, sino la confianza en sí mismo y en el mundo. Esta confianza es grabada en el cuerpo.

Cuando hay falta de adaptación inicial, ésta se congela, apareciendo fenomenológicamente como desconfianza. Ocurre entonces, la estructuración de un falso self para proteger la esperanza de una oportunidad de descongelamiento de la situación de fracaso que, a continuación, podrá ser vivida como éxito, pues de esta forma ocurre un encuentro humano.

En este caso, lo que es importante entonces es el acontecimiento, es decir, la experiencia humana relacional.

\section{Concepción salud}

Para Winnicott hablar de salud no tiene que ver con el uso de los criterios de clasificación de diagnóstico psiquiátrico. El autor relaciona salud al proceso de maduración psíquica. El individuo es saludable, en la medida en que es maduro para el momento de desarrollo que atraviesa. De un bebé se espera que pueda ser dependiente, así como de un adulto se piensa en autonomía (Tosta, 2013; Winnicott, 1967/1996b).

En el sentido que el autor le atribuye, salud no tiene que ver con la ausencia de patologías, por el contrario, el individuo sano es tolerante a la enfermedad. Al igual que en otros procesos descritos, Winnicott relaciona salud con capacidad de vivir de forma creativa.

\section{Salud (health)}

Adam Phillips en su libro contrasta sanidad y locura y afirma que hasta hoy se habla más de locura que de salud. Phillips (2008) se cuestiona:

Sabemos dónde encontrar los locos, y con quién deberíamos hablar de ellos (psiquiatras, genetistas, neurobiólogos, psicoanalistas, químicos, antropólogos, historiadores, etc.). Pero ¿dónde podemos ir para encontrar los sanos? ¿En qué edificio viven? ¿Cómo se visten? ¿Cómo son y cómo podemos reconocerlos? (p. 31)

Más adelante, él responde: "los sanos, sean quienes sean y estén donde estén, nunca han recibido la atención y el interés que merecen” (p. 31). 


\section{ARTIGOS}

El Psicoanálisis ayudó a pensar de forma más compleja la relación entre sanidad y locura.

José Outeiral (2013a) en su texto "Living creatively" toma ideas de Winnicott para diferenciar salud (health) de salud (sanity). El autor nos recuerda que Freud, en un sentido amplio, considera al individuo "sano" como aquel que es capaz de amar y trabajar; y Winnicott añade a este concepto la capacidad de vivir de forma creativa.

\section{Salud y procesos de maduración}

Winnicott (1967/1996b) en un texto que busca introducir su forma de pensar acerca de lo que sería un individuo "sano", acentúa que esta caracterización es difícil porque depende de un proceso de desarrollo y también de qué aspecto está siendo examinado. Por ejemplo, salud en la vida precoz no puede ser pensada como individual: hay que pensar en la salud de la pareja relacional, en la mayoría de los casos, resultando en la salud madre-bebé. Además, podremos focalizar la salud en relación con el desarrollo de la libido, y en este sentido, se habla de:

(...) la libertad dentro de la personalidad, de la capacidad para tener confianza y

fe, de cuestiones de constancia y confiabilidad objetal, de libertad en relación a la auto-ilusión, y también de algo que tiene que ver más con la riqueza que con la pobreza en cuanto calidad de la realidad psíquica personal. (p. 21)

En cuanto a los procesos de maduración, foco de su teoría, el autor retoma sus ideas con respecto a la constitución del ego, explayando la idea de que para cada una de las tareas iniciales del ser humano se constituye un aspecto de salud. Así habla de integración, personalización, el contacto con la realidad, y de la relación del individuo con la sociedad.

La integración es la tarea fundamental: convertirse en una unidad, el Yo soy, y así sucesivamente, pero la desintegración vivida en el estado de descanso, relajación y sueño, también es parte de la vida sana y es una condición para la creatividad. Habitar su propio cuerpo y tener placer en el uso del cuerpo y de sus funciones también está asociado con la salud psíquica, así como el uso del intelecto como ornamento del psiquismo y no como un sustituto de la asociación psicosomática.

En cuanto a las relaciones objetales, Winnicott (1967/1996b) afirma:

Una gran parte de la vida sana tiene que ver con las diferentes modalidades de relación objetal y con un proceso de "ir y venir" entre la relación con los objetos internos y externos. 
(...) La salud incluye aquí la idea de una vida emocionante y de la magia de la intimidad. (...)

La consecuencia es que el mundo interno de la persona sana se relaciona con el mundo real o externo, y sin embargo es personal y tiene su propia vivacidad. (p. 24)

Cabe señalar que en este ir y venir entre objetos internos y externos balancean los bordes de la liviana cortina de la transicionalidad que vienen del impulso creativo. Aquí la salud tiene un parentesco con la precariedad del juego, a diferencia de la rigidez de la vida defendida.

\section{Salud y diversos tipos de personas}

En varios textos, Winnicott nos recuerda de las distintas formas de organización psíquica y en el texto de 1967/1996b habla de ellas en relación con la salud.

En el primer grupo, las personas que han tenido un buen inicio relacional tienden a la salud, o las que han sido traumatizadas tienden a llevar una vida tensa, pero tal vez con salud.

En un segundo grupo, están las que se organizaron rígidamente, y por lo tanto, se distancian de la vida saludable.

Winnicott (1961/1994c) dice que "la persona sana está relativamente libre de la represión masiva y de la inhibición del instinto. (...) clínicamente, la persona realmente saludable está más cerca de la depresión y de la locura que de la psiconeurosis" (p. 58; énfasis del autor). Pienso que donde puede existir la convivencia con las artes y / o con la zona intermedia de la vida humana, puede haber salud.

El tercer grupo es paradójico: son personas que han tenido la experiencia de ansiedades impensables y construyeron una organización de defensa contra tales ansiedades, pero pueden utilizar las oportunidades de enfermarse y acercarse de lo sucedido y no vivido. Complementa diciendo que "a veces el colapso conduce a un tipo de curación, y luego vuelve a aparecer la palabra salud" (Winnicott, 1967/1996b, p. 25, énfasis mío)

En otro texto de 1967, dedicado al concepto de regresión clínica, Winnicott dice que la enfermedad del paciente comporta un elemento sano, cuando se relaciona a la regresión, a la dependência. Dice: "Una cosa es si un paciente simplemente se ha colapsado, y otra si lo tiene en algún nuevo entorno que proporciona una atención confiable" (1967/1994a, p. 154).

Salud y fuga hacia la sanidad 


\section{ARTIGOS}

Winnicott (1967/1996b) subraya que la fuga hacia la sanidad no es salud, porque la persona sana es tolerante hacia la enfermedad. Hay una amplia gama de personas sobre adaptadas que aparentan ser sanas, pero que carecen de la vivencia de la precariedade, de la creatividad y de la espontaneidad.

Muchos organizan un falso self para proteger al verdadero self, y el costo de esta adaptación sumisa es el distanciamiento del centro creativo.

Al final, sólo viviendo el self es que cada persona puede experimentar el ser y el hacer. Winnicott (1967/1996b) afirma: "ser y sentirse real se asocian a salud (...) hay un vínculo entre la salud emocional individual y el sentimiento de sentirse real"' (p. 27).

\section{Mi tesis}

A la salud que es rescatada en la consulta terapêutica, lhamo salud potencial. Postulo que esta reserva de salud, salud potencial, es lo que puede ser relacionado al denominado fenómeno curativo.

Fenómeno curativo: healing phenomena

Outeiral (2012) me despertó a esta cuestión en sus comunicaciones en el Acontecimiento previo al VII Encuentro Brasileño sobre el Pensamiento de D. W. Winnicott, del mismo año, al comentar el caso de Robin. El psicoanalista se preguntó: “¿Cómo puede una entrevista poner en marcha el desarrollo?” Su respuesta destaca el fenómeno curativo, en el original, healing phenomena.

Dice Outeiral (2013b), que en inglés la palabra cura es de afuera hacia adentro, puede ser una interpretación, un remedio, un abrazo. Mientras que el término to heal se refiere a algo que viene de adentro hacia afuera como la cicatrización emocional.

Antes Winnicott, en el texto "A cura" de 1970/1996a, cuestiona el sentido que la palabra cura adquirió en la práctica médica como la erradicación del mal o de la enfermedad. Cree que en su origen significaba cuidado. A partir de empatizar con la expectativa que tiene una persona al buscar atención médica, y sobre todo por las experiencias que tuvo en Psicoanálisis, puede llegar a la propuesta de cuidar-curar, que considera una extensión del concepto de holding. Se relaciona con la postura del médico o terapeuta en atender a las necesidades de los pacientes, permitiendo el desarrollo.

El autor propone que se encuentre el aspecto cuidar-curar en el trabajo profesional reeditando el cuidado recibido en el inicio de la vida. Y recuerda que el trabajo terapéutico se une a los fenómenos naturales y a los universales 
que "nos encontramos en la mejor poesía, filosofías y religiones" (Winnicott, 1970/1996a, p. 93).

Por lo tanto, la atención como forma de trabajo profesional se relaciona con la activación de los mecanismos de curación del paciente.

Winnicott conceptúa el fenómeno curativo - healing phenomena - en el texto redactado en 1954/1993, cuando trata del manejo de la regresión en el setting analítico. En la regresión a la dependência, se ofrece la oportunidad de encuentro humano en el que la esperanza puede ser activada y, por lo tanto, la situación de fracaso precoz puede ser descongelada y re-experimentada, esta vez en un entorno adecuado.

El autor señala también que el descongelamiento del fracaso puede ocurrir en situaciones no caracterizadas como dentro de un contexto terapéutico, mencionando "fenómenos de la vida cotidiana que tienen un poder de curación, a saber, las amistades, cuidados proporcionados durante una enfermedad física, la poesía etc." (Winnicott, 1954/1993, pp. 466-467; énfasis mío).

De acuerdo con el autor, los healing phenomena provienen de recuerdos muy precoces de cuidados cuando éramos muy pequeños. Son recuerdos de un período pre-verbal, es decir, anterior a la representación de la palabra, no simbólica, por eso Winnicott lo llama "catalogación": registro de eventos.

El autor concluye diciendo que este estudio le mostró:

(...) una nueva comprensión del 'self verdadero' y del 'self falso', del 'ego observador' como también la organización del ego que permite que la regresión sea un mecanismo de curación, el cual permanece potencial, salvo que se disponga una adaptación ambiental nueva y fiable, que pueda ser utilizada por el paciente para corregir el fracaso adaptativo original. (Winnicott, 1954/1993, p. 480; énfasis mío)

Tomemos un breve relato de uno de los casos más conocidos de las “Consultas Terapéuticas" de Winnicott (1971/1984): el caso Iiro. Elegí este caso para mantener la atención en las posibles asociaciones con el fenómeno curativo y no en la novedad de cualquier otro caso.

Se trata de un niño de 9 años y 9 meses, en la época, que sufría de sindactilia ${ }^{1}$ y fue tratado en un hospital en Finlandia. A pesar de que no era un caso que requería una atención psicoterapéutica, había un malestar en el equipo ortopédico que lo trataba, ya que había mucha complacencia del niño

${ }^{1}$ La sindactilia es una anormalidad embriológica que resulta en unión visible entre dos o más dedos de manos y pies. 


\section{ARTIGOS}

y de la madre con las numerosas cirugías de corrección, lo que es poco común en los casos en los que se requieren de cirugía. Winnicott subraya que la barrera del lenguaje - fueron traducidos por un intérprete - no interfirió en la relación que se pudo establecer entre él y el niño.

Aquí ya vemos uno de los elementos que se producen en este tipo de relación que propicia el fenómeno curativo, que es la comunicación directa entre el terapeuta y el paciente. Como un medio para establecer una comunicación profunda con los niños, fomentada por el lenguaje intermedio lúdico, que es la comunicación directa entre el terapeuta y el paciente.

Winnicott propone lo que llama el juego de trazos. Juego sin reglas ideado por Winnicott (1968/1994d) a ser utilizado para establecer la comunicación con el niño de forma rápida. En este, el consultor hace un trazo y el niño completa y luego le toca al niño hace trazos, y el profesional completa el diseño.

Entonces, con lápiz y papel, invita a Iiro a este juego. Al completar el primer trazo, el niño hace un pie de un pato. La referencia inmediata a su incapacidad sorprendió al consultor que esperó otras producciones para trabajar con esta presentación de la cuestión. Esto se refiere a la afirmación de Winnicott (1965/1994b) de que, cuando existen condiciones de setting y de postura profesional adecuada, si el niño tiene un sufrimiento este lo expresará.

Volviendo, los "dibujos" se fueron sucediendo y el terapeuta espera y registra mentalmente lo que ocurre hasta tener la certeza de que el niño esté preparado para tratar la cuestión fundamental que trae, en el caso de Iiro el sufrimiento en relación a su propia discapacidad. Llega un momento en la consulta cuando el consultor percibe una reticencia del niño para mirar sus propias manos, pero espera y no dice nada. Entonces en el dibujo número 11, el niño hace una mano deforme. Frente a la pregunta de Winnicott (1971/1984, p. 27) de que estaría pensando cuando dibujó, el niño dice: “"Pasó no más'. Y el mismo se sorprendió".

Observen que la consulta está siendo atravesada por la espera y la sorpresa del analista y del paciente. La espera del analista muestra que él se encuentra en una posición de holding, dentro de una situación de manejo clínico y no se centra en la tarea de interpretar. Su sorpresa revela que no tiene una idea preconcebida sobre el niño o el proceso, siendo también algo nuevo para el terapeuta cuando sucede. La sorpresa del paciente indica la superación de una disociación cuando ocurre lo nuevo en la consulta: algo que no estaba bajo el dominio del yo pasa a estar. 
Continúan dibujando hasta que el niño habla directamente de su cuestión, relatando que ya había hecho muchas cirugías, que aún habrían más y que sus pies continuaban iguales. Por lo tanto, el consultor puede referirse a los patos y al hecho de que las cirugías alteran la forma de cómo nació. El niño también reveló deseos de ser flautista y trabajar con las cosas que su condición hacía imposible.

El proceso de comunicación fue evolucionando hasta que Iiro dibujó una anguila lo que el autor piensa que es la manifestación del estado primitivo del niño y fantasía de pre-nacimiento. Sólo entonces, el consultor realizó una intervención verbal integradora, diciendo que Iiro se quería de pequeño, cuando podía nadar en los lagos como los patos. Y Winnicott (1971/1984) añade: "Ya crecido, deseas tocar el piano, la flauta y hacer trabajos manuales, y por lo tanto estás de acuerdo en seguir con las operaciones, pero lo más importante es ser amado tal como eres y de la forma en que naciste" (p. 31).

Iiro replicó diciendo que la madre era como él, al que el autor piensa que el chico tuvo que hacer frente a la condición de incapacidad de su madre ${ }^{2}$ bien como ala suya misma. Ellos hicieron más algunos dibujos, y el último trazo, que el autor afirma que hizo complejo, Iiro completó diciendo: "Es un pato". Winnicott (1971/1984) por lo tanto pudo concluir que el niño estaba "reafirmando su amor por sí mismo, lo que indica que él se sintió amado" (p. 34). Enfatizó la necesidad que Iiro tuvo de ser amado de la forma en que nació.

Revela allí, cual fue la comunicación significativa de la consulta y el amago de la angustia del niño.

En esta consulta, creo que cuando Winnicott enfatiza la cuestión de ser amado tal como es, se refiere a la constitución del self personal, formado a partir del espejamiento en el rostro materno. Poder vivir el self verdadero tiene que ver con la aceptación inicial del otro ser humano dedicado. La ocurrencia del fenómeno curativo en la consulta tiene que ver con la reactivación del verdadero self.

El autor señala que la relación terapéutica en las consultas terapéuticas es diferente de la situación clínica habitual de análisis. Una de las características importantes es que el analista es puesto en una posición subjetiva por el paciente y utiliza el material relativamente poco defendido que el paciente trae en esa condición. Por lo tanto recomienda evitar estimular y trabajar con

${ }^{2}$ Después el autor cuenta la entrevista que hizo la madre de Iiro y que también tuvo un efecto terapéutico. 


\section{ARTIGOS}

la transferencia, así como el uso de interpretaciones. Si el material proviene de forma más subjetiva, entonces es por el propio setting de las consultas, por el manejo y por el cuidado terapéutico, que se procesa el trabajo clínico. El conjunto de esta situación favorece la creación de un espacio donde el ser personal de los participantes pueda emerger.

Detallando las ideas, Winnicott resalta, cuando conceptualiza las consultas terapéuticas, que en ellas el profesional se ve como objeto subjetivo ${ }^{3}$ del paciente. Afirma que es típico que el paciente ponga al terapeuta en una posición especial dentro de su imaginario. Es frecuente incluso que el paciente sueñe con él la noche antes de la consulta. Se puede decir que el terapeuta fue creado por el paciente dentro de su mundo subjetivo (Tosta, 2012; Winnicott, 1971/1984).

Sería análogo al proceso de ilusión, en el que el bebé crea como objeto subjetivo el seno que, en realidad, está ahí para ser encontrado. Se puede decir que el objeto subjetivo es el objeto concebido por el sujeto. "Existe una superposición entre lo que la madre suministra y lo que el niño podría concebir" (Winnicott, 1971/1975, p. 27).

Para Gilberto Safra (1999), "el objeto subjetivo es resultado de la creatividad primaria y de la omnipotencia del bebé, posibilitando el surgimiento de la experiencia de ilusión que funda el acontecer humano" (p. 99).

\section{Consideraciones finales}

Enfin, podemos relacionar el fenómeno curativo con los recuerdos de los intercambios humanos precoces. Son registros de cuidados archivados en el psicosoma. Sería, pues, el quantum de la salud personal.

En las consultas lo que se moviliza es esta clase de fenómeno. Entonces estamos postulando que este poder de curación, este potencial de salud, también es accionado en las consultas terapéuticas, así como en las relaciones humanas significativas.

${ }^{3}$ En las palabras de Winnicott (1971/1975): "El seno es creado por el bebé repetidas veces, por la capacidad que tiene de amar o por la necesidad. Se desarrolla un fenómeno subjetivo, al que llamamos seno de la madre. La madre coloca el seno real exactamente donde el bebé está listo para crearlo, y en el momento exacto" (p. 26). 
Tanto en el manejo de la regresión a la dependencia durante el análisis, como en las consultas terapêuticas, el setting, es decir la situación establecida y su gestión, son esenciales en detrimento de la interpretación. Esto porque no se trata de representación, sino de la presentación del self que se hace presente. Así que lo importante es vivir una nueva experiencia, el evento que sorprende, y que ocurre en la relación intersubjetiva.

Dada la oportunidad terapéutica de la dependencia, hay espacio para este tipo de experiencia que revela el poder de curación del paciente, sea adulto o niño. Este mecanismo de curación puede estar relacionado con la esperanza del paciente, al despertar de su poder curativo.

La consulta puede ser un espacio de rescate de salud en el que lo que estaba escondido del propio paciente puede ser encontrado en presencia de otro ser humano.

\section{Referencias}

Outeiral, J. (2012,agosto). Comunicação oral. Evento Pré-Encontro do "VII Encontro Brasileiro sobre o Pensamento de D.W.Winnicott", Sedes Sapientiae, São Paulo.

Outeiral, J. O. (2013a). Living creatively: the concept of a sound-minded individual and the healing phenomena. In G. Goldstein (Ed.), Art in Psychoanalysis: A Contemporary Approach to Creativity and Analytic Practice. EUA: Stylus Public Ed.

Outeiral, J. O. (2013b). O conceito de indivíduo são: o viver criativo e os fenômenos curativos. Rabisco: Revista de Psicanálise. III(2), 355-363.

Phillips, A. (2008). Louco para ser normal. Rio de Janeiro, RJ: Jorge Zahar.

Safra, G. (1999, junho). A clínica em Winnicott. Natureza Humana. I(1), 91-101.

Tosta, R. M. (2012). Os princípios das consultas terapêuticas como parâmetros para a clínica winnicottiana. In J. Outeiral et. al. (Orgs.), Winnicott: Seminários Cearenses. São Paulo, SP: Zagodoni.

Tosta, R. M. (2013, outubro). Intervenção psicoprofilática com bebês doentes e seus país: Saúde x Trauma. II Encontro Internacional e IX Encontro Nacional sobre o Bebê. Brasília, DF.

Winnicott, D. W. (1975). O brincar e a realidade. Rio de Janeiro, RJ: Imago. (Trabajo original publicado em 1971).

Winnicott, D. W. (1984).Consultas terapêuticas em psiquiatria infantil. Rio de Janeiro, RJ: Imago. (Trabajo original publicado en 1971).

Winnicott, D. W. (1990). Comunicação e falta de comunicação levando ao estudo 


\section{ARTIGOS}

de certos opostos. In $O$ ambiente e os processos de maturação: estudos sobre a teoria do desenvolvimento emocional (3a ed., pp. 163-174). Porto Alegre, RS: Artes Médicas. (Trabajo original publicado en1963).

Winnicott, D. W. (1993). Aspectos clínicos e metapsicológicos da regressão dentro do setting psicanalítico. In Textos Selecionados: Da Pediatria à Psicanálise (pp. 459-481). Rio de Janeiro, RJ: Francisco Alves. (Trabajo original publicado en 1954).

Winnicott, D. W. (1994a). O conceito de regressão clínica comparado com o de organização defensiva. In C.Winnicott, R.Shepherd, \& M. Davis (Orgs.), Explorações psicanalíticas (pp. 151-156). Porto Alegre, RS: Artes Médicas Sul. (Trabajo original publicado en 1967).

Winnicott, D. W. (1994b). O valor da consulta terapêutica. In C. Winnicott, R. Shepherd, \& M. Davis (Orgs.), Explorações psicanalíticas (pp. 244-248). Porto Alegre, RS: Artes Médicas Sul. (Trabajo original publicado en 1965).

Winnicott, D. W. (1994c). Psiconeurose na infância. In C. Winnicott, R. Shepherd, \& M. Davis (Orgs.), Explorações Psicanalíticas (pp. 53-58). Porto Alegre, RS: Artes Médicas Sul. (Trabajo original publicado en 1961).

Winnicott, D. W. (1994d). O jogo do rabisco [Squiggle Game]2. In C. Winnicott, R. Shepherd, \& M. Davis (Orgs.), Explorações Psicanalíticas (pp. 230-243). Porto Alegre, RS: Artes Médicas Sul. (Trabajo original publicado en 1968).

Winnicott, D. W. (1996a). A cura. In Tudo começa em casa (pp. 87-93). São Paulo, SP: Martins Fontes. (Trabajo original publicado en 1970).

Winnicott, D. W. (1996b). O conceito de indivíduo saudável. In Tudo começa em casa (pp. 17-30). São Paulo, SP: Martins Fontes. (Trabajo original publicado en 1967).

\section{Resumo}

(Consultas terapêuticas: fenômenos curativos e saúde)

Neste trabalho relaciono os conceitos de saúde e de fenômenos curativos de D. W. Winnicott com sua proposta de consultas terapêuticas, modalidade de atendimento que ativa a criatividade da dupla psicanalista e paciente. A ideia pressupõe que o ser humano tem capacidades que emergem mesmo em situações muito críticas que perpassam a existência humana. Nas consultas, é comum que o paciente coloque o terapeuta me posição especial dentro de seu imaginário, possibilitando um tipo de relação subjetiva, diferente da transferência que se efetua em um processo analítico tradicional.

Palavras-chave: Consulta terapêutica, fenômenos curativos, saúde, criatividade 
(Therapeutic consultations: healing phenomena and health)

In this paper, I relate the concepts of health and healing phenomena of D.W. Winnicott to his proposition of therapeutic consultations, a clinical service approach that activates the creativity of both psychoanalyst and patient. The assumption is that human beings possess capabilities that emerge even facing the extremely critical situations that are a part of human existence. In these consultations, patients commonly place therapists in a special place in their imagination, allowing for a subjective relationship that differs from the transference that takes place within a traditional analytical process.

Key words: Therapeutic consultation, healing phenomena, health, creativity

(Consultations thérapeutiques: phénomènes curatifs et de santé)

Dans ce travail, j'établis une connexion entre les concepts de santé et les phénomènes de guérison de D. W. Winnicott et sa proposition des consultations thérapeutiques, un type de traitement qui active la créativité de la paire patient-psychanalyste. Je pars de l'idée que l'être humain possède des capacités qui émergent dans les situations très critiques qui traversent l'existence humaine. Au cours des consultations le patient place souvent le thérapeute dans une position particulière au sein de son imagination, donnant lieu à une sorte de relation subjective, différent du transfert qui lui a lieu dans un processus d'analyse traditionnel.

Mots clés: Consultation thérapeutique, phénomènes de guérison, santé, créativité

(Therapeutische Konsultationen: Heilphänomene und Gesundheit)

Dieser Artikel untersucht den Zusammenhang zwischen den Begriffen Gesundheit und Heilphänomen von D. W. Winnicott und seinen Ansatz der therapeutischen Konsultationen, ein Behandlungskonzept, welches die gemeinsame Entwicklung der Kreativität des Psychoanalytikers und des Patienten ermöglicht. Dieser Ansatz geht davon aus, dass der Mensch Fähigkeiten besitzt, die sich auch in sehr kritischen Situationen der menschlichen Existenz entwickeln. Ein typisches Merkmal der Konsultationen besteht darin, dass der Patient dem Therapeuten eine besondere Stelle in seiner Vorstellungswelt zuweist und somit eine Art subjektive Beziehung ermöglicht, die sich von der Übertragung des traditionellen analytischen Prozesses unterscheidet.

Schlüsselwörter: Therapeutische Konsultation. Heilphänomene. Gesundheit Kreativität

Citação/Citation: Tosta, R. M. (2017, dezembro). Consultas terapéuticas: fenómenos curativos y salud. Revista Latinoamericana de Psicopatologia Fundamental, 20(4), 762-775. http://dx.doi.org/10.1590/1415-4714.2017v20n4p762.9

Editores do artigo/Editors: Profa. Dra. Ana Maria Rudge e Profa. Dra. Sonia Leite 


\section{ARTIGOS}

Recebido/Received: 13.4.2017/ 4.13.2017 Aceito/Accepted: 28.6.2017 / 6.28.2017

Copyright: (C) 2009 Associação Universitária de Pesquisa em Psicopatologia Fundamental/ University Association for Research in Fundamental Psychopathology. Este é um artigo de livre acesso, que permite uso irrestrito, distribuição e reprodução em qualquer meio, desde que o autor e a fonte sejam citados / This is an open-access article, which permits unrestricted use, distribution, and reproduction in any medium, provided the original authors and sources are credited.

Financiamento/Funding: Apoio financeiro da PIPEQ-PUC-SP para participar do Encontro / Soutien financier du PIPEQ-PUC-SP pour participer à la réunion.

Conflito de interesses/Conflict of interest: A autora declara que não há conflito de interesses / The author has no conflict of interest to declare.

\section{Rosa Maria Tosta}

Psicoterapeuta; Doctora en Psicología Clínica; Profesora asociada de la Pontifícia Universidad Católica de Sao Paulo - PUC-SP (São Paulo, SP, Br); Profesora del curso de postgrado en Psicología Clínica y supervisora de desarrollo clínico-institucional en Psicoterapia Infantil de la Clínica PUC-SP.

Rua Monte Alegre, 984. Sala t-52.

05014-901 São Paulo, SP, Br.

romtost@pucsp.br / rosamariarmt@terra.com.br

This is an open-access article, which permits unrestricted use, distribution,

(c) BY-NC and reproduction in any medium for non-commercial purposes provided the original authors and sources are credited. 